\title{
Semi-invariant warped product submanifolds of cosymplectic manifolds
}

\author{
Meraj Ali Khan ${ }^{1 *}$, Siraj Uddin ${ }^{2}$ and Rashmi Sachdeva ${ }^{3}$
}

\author{
* Correspondence: meraj79@gmail. \\ com \\ 'Department of Mathematics, \\ University of Tabuk, Tabuk, \\ Kingdom of Saudi Arabia \\ Full list of author information is \\ available at the end of the article
}

\begin{abstract}
In this article, we obtain the necessary and sufficient conditions that the semiinvariant submanifold to be a locally warped product submanifold of invariant and anti-invariant submanifolds of a cosymplectic manifold in terms of canonical structures $T$ and $F$. The inequality and equality cases are also discussed for the squared norm of the second fundamental form in terms of the warping function. 2000 AMS Mathematics Subject Classification: 53C25; 53C40; 53C42; 53D15.
\end{abstract}

Keywords: warped product, semi-invariant submanifold, canonical structure, cosymplectic manifold

\section{Introduction}

Bishop and O'Neill [1] introduced the notion of warped product manifolds in order to construct a large variety of manifolds of negative curvature. Later on, the geometrical aspects of these manifolds have been studied by many researchers (c.f., [2-5]). The idea of warped product submanifolds was introduced by Chen [6]. He studied warped product CR-submanifolds of the form $M=M_{\perp} \times_{\lambda} M_{T}$ such that $M_{\perp}$ is a totally real submanifold and $M_{T}$ is a holomorphic submanifold of a Kaehler manifold $\bar{M}$ and proved that warped product CR-submanifolds are simply CR-products. Therefore, he considered the warped product CR-submanifolds in the form of $M=M_{T} \times_{\lambda} M_{\perp}$ which are known as CR-warped products where $M_{T}$ and $M_{\perp}$ are holomorphic and totally real submanifolds of a Kaehler manifold $\bar{M}$, respectively.

The warped product submanifolds of cosypmlectic manifolds was studied by Khan et. al [7]. Recently, Atçeken studied warped product CR-submanifolds of cosymplectic space form and obtained an inequality for the squared norm of the second fundamental form [2]. In this article, we obtain some basic results of semi-invariant submanifolds of cosymplectic manifolds and prove that a semi-invariant submanifold $M$ of a cosymplectic manifold $\bar{M}$ is locally a Riemannian product if and only if the canonical structure $T$ is parallel. The semi-invariant warped product submanifolds are the generalization of locally Riemannian product submanifolds, so it will be worthwhile to study warped product submanifolds in terms of canonical structures $T$ and $F$, to this end we obtain some characterization results on the warped product semi-invariant submanifolds in terms of the canonical structures $T$ and $F$.

(c) 2012 Khan et al.; licensee Springer. This is an open access article distributed under the terms of the Creative Commons Attribution License (http://creativecommons.org/licenses/by/2.0), which permits unrestricted use, distribution, and reproduction in any medium, provided the original work is properly cited. 


\section{Preliminaries}

A $(2 m+1)$-dimensional $C^{\infty}$-manifold $\bar{M}$ is said to have an almost contact structure if there exist on $\bar{M}$ a tensor field $\varphi$ of type $(1,1)$, a vector field $\xi$ and 1-form $\eta$ satisfying:

$$
\phi^{2}=-I+\eta \otimes \xi, \phi(\xi)=0, \eta \circ \phi=0, \eta(\xi)=1 .
$$

There always exists a Riemannian metric $g$ on an almost contact manifold $\bar{M}$ satisfying the following conditions

$$
g(\phi X, \phi Y)=g(X, Y)-\eta(X) \eta(Y), \eta(X)=g(X, \xi)
$$

where $X, Y$ are vector fields on $\bar{M}$.

An almost contact structure $(\varphi, \xi, \eta)$ is said to be normal if the almost complex structure $J$ on the product manifold $\bar{M} \times R$ is given by

$$
J\left(X, f \frac{d}{d t}\right)=\left(\phi X-f \xi, \eta(X) \frac{d}{d t}\right)
$$

where $f$ is the $C^{\infty}$-function on $\bar{M} \times R$ has no torsion i.e., $J$ is integrable. The condition for normality in terms of $\varphi, \xi$, and $\eta$ is $[\varphi, \varphi]+2 d \eta \otimes \xi=0$ on $\bar{M}$, where $[\varphi, \varphi]$ is the Nijenhuis tensor of $\varphi$. Finally, the fundamental two-form $\Phi$ is defined by $\Phi(X$, $Y)=g(X, \varphi Y)$.

An almost contact metric structure $(\varphi, \xi, \eta, g)$ is said to be cosymplectic, if it is normal and both $\Phi$ and $\eta$ are closed [8], and the structure equation of a cosymplectic manifold is given by

$$
\left(\bar{\nabla}_{X} \phi\right) Y=0
$$

for any $X, Y$ tangent to $\bar{M}$, where $\bar{\nabla}$ denotes the Riemannian connection of the metric $g$ on $\bar{M}$. Moreover, for cosymplectic manifold

$$
\bar{\nabla}_{X} \xi=0 .
$$

Let $M$ be a submanifold of an almost contact metric manifold $\bar{M}$ with induced metric $g$ and if $\nabla$ and $\nabla^{\perp}$ are the induced connections on the tangent bundle $T M$ and the normal bundle $T^{\perp} M$ of $M$, respectively. Denote by $\mathcal{F}(M)$ the algebra of smooth functions on $M$ and by $\Gamma(T M)$ the $\mathcal{F}(M)$-module of smooth sections of a vector bundle $T M$ over $M$, then the Gauss and Weingarten formulae are given by

$$
\begin{aligned}
& \bar{\nabla}_{X} Y=\nabla_{X} Y+h(X, Y) \\
& \bar{\nabla}_{X} V=-A_{V} X+\nabla_{X}^{\perp} V
\end{aligned}
$$

for each $X, Y \in \Gamma(T M)$ and $V \in \Gamma\left(T^{\perp} M\right)$, where $h$ and $A_{V}$ are the second fundamental form and the shape operator (corresponding to the normal vector field $V$ ) respectively, for the immersion of $M$ into $\bar{M}$. They are related by

$$
g(h(X, Y), V)=g\left(A_{V} X, Y\right),
$$

where $g$ denotes the Riemannian metric on $\bar{M}$ as well as on $M$. The mean curvature vector $H$ on $M$ is given by 


$$
H=\frac{1}{n} \sum_{i=1}^{n} h\left(e_{i}, e_{i}\right)
$$

where $n$ is the dimension of $M$ and $\left\{e_{1}, e_{2}, \ldots, e_{n}\right\}$ is a local orthonormal frame of vector fields on $M$. The squared norm of the second fundamental form is defined as

$$
\|h\|^{2}=\sum_{i, j=1}^{n} g\left(h\left(e_{i}, e_{j}\right), h\left(e_{i}, e_{j}\right)\right)
$$

For any $X \in \Gamma(T M)$, we write

$$
\phi X=T X+F X
$$

where $T X$ and $F X$ are the tangential and normal components of $\varphi X$, respectively.

Similarly, for any $V \in \Gamma\left(T^{\perp} M\right)$, we write

$$
\phi V=t V+f V,
$$

where $t V$ is the tangential component and $f V$ is the normal component of $\varphi V$. The covariant derivatives of the tensors $T$ and $F$ are defined as

$$
\begin{aligned}
& \left(\bar{\nabla}_{X} T\right) Y=\nabla_{X} T Y-T \nabla_{X} Y \\
& \left(\bar{\nabla}_{X} F\right) Y=\nabla_{X}^{\perp} F Y-F \nabla_{X} Y
\end{aligned}
$$

for all $X, Y \in \Gamma(T M)$.

Let $M$ be a Riemannian manifold isometrically immersed in an almost contact metric manifold $\bar{M}$, then for every $x \in M$ there exist a maximal invariant subspace denoted by $D_{x}$ of the tangent space $T_{x} M$ of $M$. If the dimension of $D_{x}$ is same for all values of $x \in M$, then $D_{x}$ gives an invariant distribution $D$ on $M$.

A submanifold $M$ of an almost contact metric manifold $\bar{M}$ is called a semi-invariant submanifold if there exist on $M$ a differentiable distribution $D$ whose orthogonal complementary distribution $D^{\perp}$ is anti-invariant, i.e.,

(i) $T M=D \oplus D^{\perp} \oplus\langle\xi\rangle$

(ii) $D$ is an invariant distribution

(iii) $D^{\perp}$ is an anti-invariant distribution i.e., $\varphi D^{\perp} \subseteq T^{\perp} M$.

A semi-invariant submanifold is anti-invariant if $D_{x}=\{0\}$ and invariant if $D_{x}^{\perp}=\{0\}$ respectively, for every $x \in M$. It is a proper semi-invariant submanifold if neither $D_{x}=$ $\{0\}$ nor $D_{x}^{\perp}=\{0\}$, for each $x \in M$.

Let $M$ be a semi-invariant submanifold of an almost contact metric manifold $\bar{M}$. Then, $F T_{x} M$ is a subspace of $T_{x}^{\perp} M$ such that

$$
T_{x}^{\perp} M=F T_{x} M \oplus v_{x}
$$

where $v$ is the invariant subspace of $T^{\perp} M$ under $\varphi$.

Let $M$ be a proper semi-invariant submanifold of an almost contact metric manifold $\bar{M}$, then for any $X \in \Gamma(T M)$, we have

$$
X=P_{1} X+P_{2} X+\eta(X) \xi,
$$


where $P_{1}$ and $P_{2}$ are the orthogonal projections from $T M$ to $D$ and $D^{\perp}$, respectively. It follows immediately that

$$
(a) T P_{2}=0,(b) F P_{1}=0,(c) t\left(T^{\perp} M\right)=D^{\perp},(d) f T^{\perp} M \subseteq v .
$$

From (2.3), (2.5), (2.6), (2.8), and (2.9), we have

$$
\begin{aligned}
& \left(\bar{\nabla}_{X} T\right) Y=A_{F Y} X+\operatorname{th}(X, Y) \\
& \left(\bar{\nabla}_{X} F\right) Y=f h(X, Y)-h(X, T Y)
\end{aligned}
$$

for any $X, Y \in \Gamma(T M)$.

Definition 2.1 A semi-invariant submanifold $M$ is said to be a locally semi-invariant product submanifold if $M$ is locally a Riemannian product of the leaves of distributions $D, D^{\perp}$, and $\langle\bar{\zeta}\rangle$.

Definition 2.2 Let $\left(N_{1}, g_{1}\right)$ and $\left(N_{2}, g_{2}\right)$ be two Riemannian manifolds with Riemannian metrics $g_{1}$ and $g_{2}$, respectively, and $\lambda$ be a positive differentiable function on $N_{1}$. Then the warped product of $N_{1}$ and $N_{2}$ is the Riemannian manifold $\left(N_{1} \times N_{2}, g\right)$, where

$$
g=g_{1}+\lambda^{2} g_{2}
$$

The warped product manifold $\left(N_{1} \times N_{2}, g\right)$ is denoted by $N_{1} \times \lambda N_{2}$. If $U$ is any vector field tangent to $M=N_{1} \times_{\lambda} N_{2}$ at $(p, q)$, then

$$
\|U\|^{2}=\left\|d \pi_{1} U\right\|^{2}+\lambda^{2}(p)\left\|d \pi_{2} U\right\|^{2},
$$

where $\pi_{1}$ and $\pi_{2}$ are the canonical projections of $M$ onto $N_{1}$ and $N_{2}$, respectively.

Bishop and O'Neill [1] proved the following results:

Theorem 2.1 Let $M=N_{1} \times_{\lambda} N_{2}$ be a warped product manifold. If $X, Y \in \Gamma\left(T N_{1}\right)$ and $Z, W \in \Gamma\left(T N_{2}\right)$, then

(i) $\nabla_{X} Y \in \Gamma\left(T N_{1}\right)$

(ii) $\nabla_{X} Z=\nabla_{Z} X=\left(\frac{X \lambda}{\lambda}\right) Z$,

(iii) $\nabla_{Z} W=\nabla_{Z}^{N_{2}} W-\frac{g(Z, W)}{\lambda} \nabla \lambda$.

where $\nabla^{N_{2}}$ is the connection on $N_{2}$ and $\nabla \lambda$ is the gradient of the function $\lambda$ and is defined as

$$
g(\nabla \lambda, U)=U \lambda,
$$

for each $U \in \Gamma(T M)$.

Corollary 2. 1 On a warped product manifold $M=N_{1} \times_{\lambda} N_{2}$, we have

(i) $N_{1}$ is totally geodesic in $M$,

(ii) $N_{2}$ is totally umbilical in $M$.

\section{Some basic results on semi-invariant submanifolds}

In the following section, we discuss some basic results on semi-invariant submanifolds of a cosymplectic manifold for later use. First, we obtain the integrability conditions of involved distributions in the definition of a semi-invariant submanifold and then we will see the geometric properties of their leaves.

Proposition 3.1 [9] Let $M$ be a semi-invariant submanifold of a cosymplectic manifold then the anti-invariant distribution $D^{\perp}$ is integrable. 
Proposition 3.2 The invariant distribution $D$ on a semi-invariant submanifold of a cosymplectic manifold is integrable if and only if

$$
g(h(X, \phi Y), \phi Z)=g(h(\phi X, Y), \phi Z)
$$

for each $X, Y \in \Gamma(D)$ and $Z \in \Gamma\left(D^{\perp}\right)$.

Proof. The result can be obtained by making use of (2.2), (2.3), and (2.5).

Proposition 3.3 If the invariant distribution $D$ on a semi-invariant submanifold $M$ of a cosymplectic manifold $\bar{M}$ is integrable, then its leaves are totally geodesic in $M$ if and only if

$$
h(U, Y) \in \Gamma(v),
$$

for each $U \in \Gamma(T M)$ and $Y \in \Gamma(D)$.

Proof. From (2.16), we obtain

$$
F \nabla_{U} Y=f h(U, Y)-h(U, T Y),
$$

for any $U \in \Gamma(T M)$ and $Y \in \Gamma(D)$. Taking the inner product with $\varphi Z$ for any $Z \in$ $\Gamma\left(D^{\perp}\right)$, we get

$$
g\left(F \nabla_{U} Y, \phi Z\right)=-g(h(U, T Y), \phi Z) .
$$

The result follows from the above equation.

Now, we have the following corollary for later use.

Corollary 3.1 The invariant distribution $D$ on a semi-invariant submanifold $M$ of a cosymplectic manifold $\bar{M}$ is integrable and its leaves are totally geodesic in $M$ if and only if

$$
\left(\bar{\nabla}_{X} T\right) Y=0 .
$$

for any $X, Y \in \Gamma(D)$.

Proof. The result follows from (2.15) and Proposition 3.3.

Lemma 3.1 For a semi-invariant submanifold $M$ of a cosymplectic manifold $\bar{M}$, the leaf $N_{\perp}$ of $D^{\perp}$ is totally geodesic in $M$ if and only if

$$
g(h(X, Z), \phi W)=0,
$$

for any $X \in \Gamma(D)$ and $Z, W \in \Gamma\left(D^{\perp}\right)$.

Proof. From (2.2), (2.3), (2.5), and (2.6), we obtain

$$
g\left(\nabla_{Z} W, \phi X\right)=g(h(X, Z), \phi W) .
$$

Thus, the result follows from the above equation.

Theorem 3.1 A semi-invariant submanifold $M$ of a cosymplectic manifold $\bar{M}$ is locally a semi-invariant product if and only if

$$
\left(\bar{\nabla}_{U} T\right) V=0,
$$

for any $U, V \in \Gamma(T M)$.

Proof. If $T$ is parallel then by (2.15), we have

$$
A_{F V} U=-\operatorname{th}(U, V)
$$


for any $U, V$ tangent to $M$. In particular, if $X \in \Gamma(D)$, then (3.1) gives, $\operatorname{th}(U, X)=0$, that is,

$$
A_{F Z} X=0 .
$$

for any $Z \in \Gamma\left(D^{\perp}\right)$. Thus by Proposition 3.2 and Lemma 3.1, $D$ is integrable and the leaf $N_{\perp}$ of $D^{\perp}$ is totally geodesic in $M$. Let $N_{T}$ be a leaf of $D$, now for any $X, Y \in \Gamma(D)$ and $Z \in \Gamma\left(D^{\perp}\right)$ by (3.2), we obtain $g\left(A_{\varphi Z} X, Y\right)=0$ and using (2.2), (2.3), (2.5), and (2.6), we get $g\left(\nabla_{X} \varphi Y, Z\right)=0$, which shows that leaf of $D$ is totally geodesic in $M$ and distribution $\langle\xi\rangle$ is already totally geodesic in $M$ and hence $M$ is locally a semi-invariant product.

Conversely, if $M$ is locally a semi-invariant product then $\nabla_{U} \times \in \Gamma(D)$ for any $X \in$ $\Gamma(D)$ and $U \in \Gamma(T M)$, thus by (2.15) and the Proposition 3.3, we get $\left(\bar{\nabla}_{U} T\right) Y=0$. Similarly, for any $Z \in \Gamma\left(D^{\perp}\right)$ and $U \in \Gamma(T M)$, we obtain $\nabla_{U} Z \in \Gamma\left(D^{\perp}\right)$ and then by (2.10), we get $\left(\bar{\nabla}_{U} T\right) Z=0$ and it is easy to see that $\left(\bar{\nabla}_{U} T\right) \xi=0$. By these observations we find that $\left(\bar{\nabla}_{U} T\right) V=0$, for all $U, V \in \Gamma(T M)$, this proves the theorem completely.

\section{Semi-invariant warped product submanifolds}

Throughout this section, we denote $N_{T}$ and $N_{\perp}$ the invariant and anti-invariant submanifolds of a cosymplectic manifold $\bar{M}$, respectively. The warped product semi-invariant submanifolds of a cosymplectic manifold $\bar{M}$ are denoted by $N_{\perp} \times_{\lambda} N_{T}$ and $N_{T} \times_{\lambda} N_{\perp}$. The first type of warped products do not exist of a cosymplectic manifold in the sense of [5], here we discuss the second type of warped products and obtain some interesting results. First, we have the following lemma:

Lemma 4.1 Let $M=N_{T} \times_{\lambda} N_{\perp}$ be a warped product semi-invariant submanifold of an almost contact metric manifold $\bar{M}$. Then

$$
\begin{aligned}
& \left(\bar{\nabla}_{Z} T\right) X=(T X \ln \lambda) Z \\
& \left(\bar{\nabla}_{U} T\right) Z=g\left(P_{2} U, Z\right) T(\nabla \ln \lambda) .
\end{aligned}
$$

for any $X, Z$, and $U$ tangent to $N_{T}, N_{\perp}$, and $M$, respectively.

Proof. Let $M=N_{T} \times_{\lambda} N_{\perp}$ be a warped product submanifold of invariant and antiinvariant submanifolds of an almost contact metric manifold $\bar{M}$, then by Theorem 2.1 (ii), we have

$$
\nabla_{X} Z=\nabla_{Z} X=(X \ln \lambda) Z
$$

for $X \in \Gamma\left(T N_{T}\right)$ and $Z \in \Gamma\left(T N_{\perp}\right)$. Then, from (2.10) and (4.1), we get

$$
\left(\bar{\nabla}_{Z} T\right) X=(T X \ln \lambda) Z
$$

which proves the first part of the lemma. Now, for any $U \in \Gamma(T M)$, we have $T U \in$ $\Gamma\left(T N_{T}\right)$, therefore $\left(\bar{\nabla}_{U} T\right) Z \in \Gamma\left(T N_{T}\right)$ for any $U \in \Gamma(T M)$. Furthermore, for any $X \in$ $\Gamma\left(T N_{T}\right)$, we obtain

$$
g\left(\left(\bar{\nabla}_{U} T\right) Z, X\right)=-g\left(Z, \nabla_{U} T X\right) .
$$


Using (2.13), the above equation reduced to

$$
\begin{aligned}
g\left(\left(\bar{\nabla}_{U} T\right) Z, X\right) & =-g\left(Z, \nabla_{P_{1} U+P_{2} U+\eta(U) \xi} T X\right) . \\
& =-g\left(Z, \nabla_{P_{2} U} T X\right)+\eta(U) g\left(\nabla_{\xi} Z, T X\right) .
\end{aligned}
$$

Using (4.1), the second term of right hand side is identically zero, then the above equation takes the form

$$
\begin{aligned}
g\left(\left(\bar{\nabla}_{U} T\right) Z, X\right) & =-g\left(Z, \nabla_{P_{2} U} T X\right) \\
& =-(T X \ln \lambda) g\left(Z, P_{2} U\right) .
\end{aligned}
$$

Using (2.17), we obtain

$$
g\left(\left(\bar{\nabla}_{U} T\right) Z, X\right)=g(T \nabla \ln \lambda, X) g\left(Z, P_{2} U\right) .
$$

That is,

$$
\left(\bar{\nabla}_{U} T\right) Z=T(\nabla \ln \lambda) g\left(Z, P_{2} U\right) .
$$

This proves the lemma completely.

Theorem 4.1 A proper semi-invariant submanifold of a cosymplectic manifold $\bar{M}$ is locally a warped product semi-invariant submanifold if and only if

$$
\left(\bar{\nabla}_{U} T\right) V=(T V \mu) P_{2} U+g\left(P_{2} U, P_{2} V\right) \phi \nabla \mu,
$$

for each $U, V \in \Gamma(T M)$ and $\mu, a C^{\infty}$-function on $M$ satisfying $W \mu=0$, for each $W \in$ $\Gamma\left(D^{\perp}\right)$.

Proof. Let $M=N_{T} \times_{\lambda} N_{\perp}$ be a warped product semi-invariant submanifold of a cosymplectic manifold $\bar{M}$, then from (2.10) and (2.13), we have

$$
\left(\bar{\nabla}_{U} T\right) V=\left(\bar{\nabla}_{U} T\right) P_{1} V+\left(\bar{\nabla}_{U} T\right) P_{2} V+\eta(U)\left(\bar{\nabla}_{U} T\right) \xi .
$$

Again using (2.10) and (2.13), the above equation takes the form

$$
\left(\bar{\nabla}_{U} T\right) V=\left(\bar{\nabla}_{P_{1} U} T\right) P_{1} V+\left(\bar{\nabla}_{P_{2} U} T\right) P_{1} V+\left(\bar{\nabla}_{U} T\right) P_{2} V .
$$

Now, from Lemma 4.1, we have

$$
\left(\bar{\nabla}_{P_{2} U} T\right) P_{1} V=(T V \ln \lambda) P_{2} U
$$

and

$$
\left(\bar{\nabla}_{U} T\right) P_{2} V=g\left(P_{2} U, P_{2} V\right) T(\nabla \ln \lambda) .
$$

Substituting these values in (4.3), we obtain

$$
\left(\bar{\nabla}_{U} T\right) V=(T V \mu) P_{2} U+g\left(P_{2} U, P_{2} V\right) \phi \nabla \mu .
$$

Conversely, suppose that $M$ is a semi-invariant submanifold of a cosymplectic manifold $\bar{M}$ and (4.2) holds, then $\left(\bar{\nabla}_{X} T\right) Y=0$, for each $X, Y \in \Gamma(D)$. Then by Corollary 3.1, $D$ is integrable and each leave $N_{T}$ of $D$ is totally geodesic in $M$. Moreover, from (4.2), we have

$$
g\left(\left(\bar{\nabla}_{Z} T\right) X, W\right)=(T X \mu) g(Z, W) .
$$


for $X \in \Gamma(D)$ and $Z, W \in \Gamma\left(D^{\perp}\right)$. Using (2.3), (2.8), and (2.10), we obtain

$$
g\left(\phi \bar{\nabla}_{Z} X, W\right)=(T X \mu) g(Z, W) .
$$

That is,

$$
g\left(\bar{\nabla}_{Z} X, \phi W\right)=-(T X \mu) g(Z, W)
$$

Using cosymplectic character and (2.5), we derive

$$
g\left(\nabla_{Z} W, \phi X\right)=-(T X \mu) g(Z, W) .
$$

By (2.17), the above equation takes the form

$$
g\left(\nabla_{Z} W, \phi X\right)=g(T \nabla \mu, X) g(Z, W) .
$$

Let us assume that $N_{\perp}$ is a leaf of $D^{\perp}$ and $h^{\prime}$ is the second fundamental form of the immersion of $N_{\perp}$ into $M$, then

$$
g\left(h^{\prime}(Z, W), X\right)=g\left(\nabla_{Z} W, X\right) .
$$

Using (4.4), we get

$$
g\left(h^{\prime}(Z, W), \phi X\right)=-g(\nabla \mu, \phi X) g(Z, W)
$$

or,

$$
h^{\prime}(Z, W)=-g(Z, W) \nabla \mu .
$$

This means that $N_{\perp}$ is totally umbilical in $M$ with non vanishing mean curvature $\nabla \mu$. Also, as $W \mu=0$, for all $W \in \Gamma\left(D^{\perp}\right)$, i.e., the mean curvature vector of $N_{\perp}$ is parallel and the leaves of $D^{\perp}$ are extrinsic spheres in $M$. Hence from a result of Hiepko [10], the submanifold $M$ is locally a warped product semi-invariant submanifold of $N_{T}$ and $N_{\perp}$ with warping function $\lambda=e^{\mu}$.

Note. Theorem 4.1 is a generalization of Theorem 3.1, and shows that what is the effect on $\bar{\nabla} T$, when the submanifold is a warped product semi-invariant submanifold.

Theorem 4.2 A semi-invariant submanifold $M$ of a cosymplectic manifold $\bar{M}$ is locally a warped product semi-invariant submanifold if and only if

$$
g\left(\left(\bar{\nabla}_{U} F\right) V, \phi W\right)=-\left(P_{1} V \mu\right) g(U, W),
$$

for $U, V \in \Gamma(T M)$ and $W \in \Gamma\left(D^{\perp}\right)$, where $\mu$ is a $C^{\infty}$-function on $M$ such that $Z \mu=$ 0 , for all $Z \in D^{\perp}$.

Proof. If $M=N_{T} \times_{\lambda} N_{\perp}$ is a warped product semi-invariant submanifold of a cosymplectic manifold $\bar{M}$, then $N_{T}$ and $N_{\perp}$ are totally geodesic and totally umbilical in $M$, respectively. Moreover, we have

$$
\nabla_{X} Z=\nabla_{Z} X=(X \ln \lambda) Z \text {. }
$$

for any $X \in \Gamma(D)$ and $Z \in \Gamma\left(D^{\perp}\right)$. Now, by (2.13), we have

$$
\begin{aligned}
\left(\bar{\nabla}_{U} F\right) V & =\left(\bar{\nabla}_{P_{1} U+P_{2} U+\eta(U) \xi} F\right) V \\
& =\left(\bar{\nabla}_{P_{1} U} F\right) V+\left(\bar{\nabla}_{P_{2} U} F\right) V+\eta(U)\left(\bar{\nabla}_{\xi} F\right) V .
\end{aligned}
$$


Again, using (2.13), the above equation takes the form

$$
\begin{aligned}
\left(\bar{\nabla}_{U} F\right) V= & \left(\bar{\nabla}_{P_{1} U} F\right) P_{1} V+\left(\bar{\nabla}_{P_{1} U} F\right) P_{2} V+\eta(V)\left(\bar{\nabla}_{P_{1} U} F\right) \xi+\left(\bar{\nabla}_{P_{2} U} F\right) P_{1} V \\
& +\left(\bar{\nabla}_{P_{2} U} F\right) P_{2} V+\eta(V)\left(\bar{\nabla}_{P_{2} U} F\right) \xi+\eta(U)\left(\bar{\nabla}_{\xi} F\right) P_{1} V \\
& +\eta(U)\left(\bar{\nabla}_{\xi} F\right) P_{2} V+\eta(U) \eta(V)\left(\bar{\nabla}_{\xi} F\right) \xi .
\end{aligned}
$$

In view of (2.4), (2.5), and (2.16), the above equation reduced to

$$
\left(\bar{\nabla}_{U} F\right) V=\left(\bar{\nabla}_{P_{1} U} F\right) P_{1} V+\left(\bar{\nabla}_{P_{1} U} F\right) P_{2} V+\left(\bar{\nabla}_{P_{2} U} F\right) P_{1} V+\left(\bar{\nabla}_{P_{2} U} F\right) P_{2} V .
$$

Taking the inner product with $\varphi W$, for any $W \in \Gamma\left(D^{\perp}\right)$, we obtain

$$
\begin{aligned}
\left.g\left(\bar{\nabla}_{U} F\right) V, \phi W\right) & =g\left(\left(\bar{\nabla}_{P_{1} U} F\right) P_{1} V+\left(\bar{\nabla}_{P_{1} U} F\right) P_{2} V\right. \\
& \left.+\left(\bar{\nabla}_{P_{2} U} F\right) P_{1} V+\left(\bar{\nabla}_{P_{2} U} F\right) P_{2} V, \phi W\right) .
\end{aligned}
$$

Using (2.14), (2.16) and the fact that $P_{1} U \in \Gamma(D)$ and $P_{2} U \in \Gamma\left(D^{\perp}\right)$, for any $U \in$ $\Gamma(T M)$, then the above equation becomes

$$
\begin{aligned}
& g\left(\left(\bar{\nabla}_{U} F\right) V, \phi W\right)=g\left(f h\left(P_{1} U, P_{1} V\right), \phi W\right)-g\left(h\left(P_{1} U, T P_{1} V\right), \phi W\right) \\
&++g\left(f h\left(P_{1} U, P_{2} V\right), \phi W\right)+g\left(f h\left(P_{2} U, P_{1} V\right), \phi W\right) \\
&+g\left(f h\left(P_{2} U, P_{2} V\right), \phi W\right)-g\left(h\left(P_{2} U, T P_{1} V\right), \phi W\right) .
\end{aligned}
$$

From (2.2), the above equation becomes

$$
g\left(\left(\bar{\nabla}_{U} F\right) V, \phi W\right)=-g\left(h\left(P_{1} U, T P_{1} V\right)+h\left(P_{2} U, T P_{1} V\right), \phi W\right) .
$$

Using (2.5), we derive

$$
g\left(\left(\bar{\nabla}_{U} F\right) V, \phi W\right)=-g\left(\bar{\nabla}_{P_{1} U} \phi P_{1} V, \phi W\right)-g\left(\bar{\nabla}_{P_{2} U} \phi P_{1} V, \phi W\right) .
$$

Using the covariant differentiation property of $\varphi$ and the fact that $P_{1} V \in \Gamma(D)$ and $P_{2} V \in \Gamma\left(D^{\perp}\right)$, for any $V \in \Gamma(T M)$, then from (2.2), we obtain

$$
g\left(\left(\bar{\nabla}_{U} F\right) V, \phi W\right)=g\left(P_{1} V, \bar{\nabla}_{P_{1} U} W\right)-g\left(\bar{\nabla}_{P_{2} U} P_{1} V, W\right) .
$$

Again using (2.5), we arrive at

$$
g\left(\left(\bar{\nabla}_{U} F\right) V, \phi W\right)=g\left(P_{1} V, \nabla_{P_{1} U} W\right)-g\left(\nabla_{P_{2} U} P_{1} V, W\right) .
$$

The first term of right-hand side is zero by (4.1) and the fact that $P_{1} V \in \Gamma(D)$ and $W$ $\in \Gamma\left(D^{\perp}\right)$, thus we obtain

$$
\begin{aligned}
g\left(\left(\bar{\nabla}_{U} F\right) V, \phi W\right) & =-\left(P_{1} V \ln \lambda\right) g\left(P_{2} U, W\right) \\
& =-\left(P_{1} V \ln \lambda\right) g(U, W) \\
& =-\left(P_{1} V \mu\right) g(U, W) .
\end{aligned}
$$

Conversely, suppose that $M$ is a semi-invariant submanifold of a cosymplectic manifold satisfying (4.5), then it is easy to see that

$$
g\left(\left(\bar{\nabla}_{X} F\right) Y, \phi W\right)=0,
$$

for each $X, Y \in \Gamma(D)$ and $W \in \Gamma\left(D^{\perp}\right)$. Thus, by (2.16) we obtain

$$
g(h(X, \phi Y), \phi W)=0 .
$$


Therefore by Propositions 3.2 and 3.3, the distribution $D$ is integrable and its leaves are totally geodesic in $M$. Now for any $Z \in \Gamma\left(D^{\perp}\right)$, by (4.5), we have

$$
g\left(\left(\bar{\nabla}_{Z} F\right) X, \phi W\right)=-(X \mu) g(Z, W) .
$$

Using (2.16), we get

$$
g(h(\phi X, Z), \phi W)=(X \mu) g(Z, W) .
$$

Let $N_{\perp}$ be a leaf of $D^{\perp}$ and $h^{\prime}$ be the second fundamental form of the immersion of $N_{\perp}$ into $M$ and $\nabla^{\prime}$ is the induced connection on $N_{\perp}$, then by Gauss formula, we have

$$
\nabla_{Z} W=\nabla_{Z}^{\prime} W+h^{\prime}(Z, W) .
$$

Now for any $Z, W \in \Gamma\left(D^{\perp}\right)$ and $X \in \Gamma(D)$, by (2.3) and (2.5), we have

$$
g(h(Z, X), \phi W)=g\left(\phi X, \nabla_{Z} W\right) .
$$

From (4.7), we obtain

$$
g(h(Z, X), \phi W)=g\left(h^{\prime}(Z, W), \phi X\right) .
$$

Thus, by (4.6) and (4.8), we derive

$$
g\left(h^{\prime}(Z, W), X\right)=-(X \mu) g(Z, W) .
$$

Using (2.17), we obtain

$$
h^{\prime}(Z, W)=-g(Z, W) \nabla \mu,
$$

which implies that $N_{\perp}$ is totally umbilical in $M$ with non vanishing mean curvature vector $\nabla \mu$. Moreover, as $Z \mu=0$ for all $Z \in \Gamma\left(D^{\perp}\right)$ that is, the mean curvature is parallel on $N^{\perp}$, this show that $N_{\perp}$ is extrinsic sphere. Hence, from a result of [10], $M$ is locally a warped product submanifold.

Proposition 4.1. Let $M=N_{T} \times_{\lambda} N_{\perp}$ be a warped product semi-invariant submanifold of a cosymplectic manifold of $\bar{M}$. Then

(i) $h_{\phi D^{\perp}}(\phi X, Z)=(X \ln \lambda) \phi Z$

(ii) $g(h(\varphi X, Z), \varphi h(X, Z))=\left\|h_{v}(X, Z)\right\|^{2}$

for any $x \in \Gamma(D)$ and $Z \in \Gamma\left(D^{\perp}\right)$.

Proof. For any $X \in \Gamma(D)$ and $Z \in \Gamma\left(D^{\perp}\right)$, by Gauss formula, we have

$$
h(\phi X, Z)=\phi \nabla_{Z} X+\phi h(X, Z)-\nabla_{Z} \phi X .
$$

Using (4.1), we get

$$
h(\phi X, Z)=(X \ln \lambda) \phi Z+\phi h(X, Z)-(\phi X \ln \lambda) Z .
$$

Equating the tangential components of (4.9), we get

$$
(\phi X \ln \lambda) Z=\operatorname{th}(X, Z),
$$

Taking the inner product with $W \in \Gamma\left(D^{\perp}\right)$, we obtain

$$
g(h(X, Z), \phi W)=-(\phi X \ln \lambda) g(Z, W),
$$


or equivalently

$$
h_{\phi D^{\perp}}(X, Z)=-(\phi X \ln \lambda) \phi Z \text {. }
$$

Replacing $X$ by $\varphi X$, we obtain

$$
h_{\phi D^{\perp}}(\phi X, Z)=(X \ln \lambda) \phi Z,
$$

which proves the part (i) of proposition. Now, for the second part comparing the normal components of (4.9), we get

$$
h(\phi X, Z)=(X \ln \lambda) \phi Z+\phi h_{v}(X, Z),
$$

or,

$$
h(\phi X, Z)-\phi h_{v}(X, Z)=(X \ln \lambda) \phi Z,
$$

Taking the inner product with $\varphi h(X, Z)$, we derive

$$
g(h(\phi X, Z), \phi h(X, Z))=\left\|h_{v}(X, Z)\right\|^{2},
$$

which completes the proof.

Theorem 4.3. Let $M=N_{T} \times_{\lambda} N_{\perp}$ be a warped product semi-invariant submanifold of a cosymplectic manifold $\bar{M}$. Then

(i) The squared norm of the second fundamental form satisfies

$$
\|h\|^{2} \geq 2 q\|\nabla \ln \lambda\|^{2}
$$

where $\nabla \ln \lambda$ is the gradient of the function $\ln \lambda$ and $q$ is the dimension of $N_{\perp}$.

(ii) If the equality holds identically, then $N_{T}$ is a totally geodesic submanifold of $\bar{M}$, $N_{\perp}$ is a totally umbilical submanifold of $\bar{M}$ and $M$ is minimal.

Proof. Let $\left\{X_{1}, X_{2}, \ldots, X_{p}, X_{p+1}=\varphi X_{1}, \ldots, X_{2 p}=\varphi X_{p}, X_{2 p+1}=\xi\right\}$ be a local orthonormal frame of vector fields on $N_{T}$ and $\left\{Z_{1}, Z_{2}, \ldots, Z_{q}\right\}$ a local orthonormal frame on $N_{\perp}$. Then by definition of squared norm of mean curvature vector

$$
\begin{aligned}
\|h\|^{2}= & \sum_{i, j=1}^{2 p+1} g\left(h\left(X_{i}, X_{j}\right), h\left(X_{i}, X_{j}\right)\right) \\
& +\sum_{i=1}^{2 p+1} \sum_{r=1}^{q} g\left(h\left(X_{i}, Z_{r}\right), h\left(X_{i}, Z_{r}\right)\right) \\
& +\sum_{r, s=1}^{q} g\left(h\left(Z_{r}, Z_{s}\right), h\left(Z_{r}, Z_{s}\right)\right)
\end{aligned}
$$

or,

$$
\|h\|^{2} \geq \sum_{i=1}^{2 p} \sum_{r=1}^{q} g\left(h\left(X_{i}, Z_{r}\right), h\left(X_{i}, Z_{r}\right)\right)
$$


In view of Proposition 4.1 (i), we get

$$
\begin{aligned}
\|h\|^{2} & \geq \sum_{i=1}^{2 p} \sum_{r=1}^{q}\left(\phi X_{i} \ln \lambda\right)^{2} g\left(Z_{r}, Z_{r}\right), \\
& \geq 2 q\|\nabla \ln \lambda\|^{2} .
\end{aligned}
$$

This verifies the assertion (i). If the equality sign holds, then from (4.10) and Proposition 4.1 (i), we get

$$
h(D, D)=0, h\left(D^{\perp}, D^{\perp}\right)=0 \quad \text { and } \quad h\left(D, D^{\perp}\right) \in \Gamma\left(\phi D^{\perp}\right) .
$$

As $N_{T}$ is a totally geodesic submanifold of $M$, the first condition of (4.11) implies that $N_{T}$ is totally geodesic in $\bar{M}$. Moreover, $N_{\perp}$ is totally umbilical in $M$, the second condition of (4.11) implies that $N_{\perp}$ is totally umbilical in $\bar{M}$, and also it follows from (4.11) that $M$ is minimal in $\bar{M}$.

\section{Acknowledgements}

MAK was supported by the Research Grant 0136-1432-S, Deanship of Scientific research, (University of Tabuk, K.S.A.) and SU was supported by the Grant RG117/10AFR (University of Malaya, Malaysia).

\section{Author details}

${ }^{1}$ Department of Mathematics, University of Tabuk, Tabuk, Kingdom of Saudi Arabia ${ }^{2}$ Institute of Mathematical Sciences, Faculty of Science, University of Malaya, 50603 Kuala Lumpur, Malaysia ${ }^{3}$ School of Mathematics and Computer Applications, Thapar University, Patiala Punjab, India

\section{Authors' contributions}

MAK carried out the geometric properties of the leaves of the involved distributions and participated to find out the geometric properties of warped products. SU participated in the study of warped products and drafted the manuscript. RS participated in the proof reading of the manuscript. All authors read and approved the final manuscript.

\section{Competing interests}

The authors declare that they have no competing interests.

Received: 4 October 2011 Accepted: 31 January 2012 Published: 31 January 2012

\section{References}

1. Bishop, RL, O'Neill, B: Manifolds of negative curvature. Trans Am Math Soc. 145, 1-49 (1965)

2. Atçeken, M: Contact CR-warped product submanifolds in cosymplectic space forms. Collect Math. 62, 17-26 (2011). doi:10.1007/s13348-010-0002-z

3. Chen, BY: Geometry of warped product CR-submanifolds in Kaehler manifolds II. Monatsh Math. 134, 103-119 (2001). doi:10.1007/s006050170002

4. Hasegawa, I, Mihai, I: Contact CR-warped product submanifolds in Sasakian manifolds. Geom Dedicata. 102, 143-150 (2003)

5. Uddin, S, Khan, KA: Warped product CR-submanifolds of cosymplectic manifolds. Ricerche Mat. 60, 143-149 (2011). doi:10.1007/s11587-010-0099-0

6. Chen, BY: Geometry of warped product CR-submanifolds in Kaehler manifolds I. Monatsh Math. 133, 177-195 (2001). doi:10.1007/s006050170019

7. Khan, KA, Khan, VA, Uddin, S: Warped product submanifolds of cosymplectic manifolds. Balkan J Geom Appl. 13, 55-65 (2008)

8. Ludden, GD: Submanifolds of cosymplectic manifolds. J Diff Geom. 4, 237-244 (1970)

9. Shoeb, M, Shahid, MH, Sharfuddin, A: On submanifolds of a cosymplectic manifold. Soochow J Math. 27, 161-174 (2001)

10. Hiepko, S: Eine Inner Kenneichung der verzerrten Produkte. Math Ann. 241, 209-215 (1979). doi:10.1007/BF01421206

doi:10.1186/1029-242X-2012-19

Cite this article as: Khan et al:: Semi-invariant warped product submanifolds of cosymplectic manifolds. Journal of Inequalities and Applications 2012 2012:19. 